\title{
Genetic patterns for adaptive evolution of TTX in Nassarius
}

\author{
Shanmei Zou ${ }^{1}$ \\ ${ }^{1}$ Nanjing Agricultural University
}

August 12, 2020

\begin{abstract}
Understanding the genetic basis of tetrodotoxin (TTX) accumulation and resistance in animals could provide us significant insights into adaptive evolution in ecological communities. It has been reported that TTX resistance is possibly due to gene mutation in sodium channels. Eating sea snail Nassarius has caused serious people poisoning and death incident due to tetrodotoxin (TTX) accumulation in their body. Here we conducted transcriptome analysis for both toxic and non-toxic communities in two Nassarius species (Nassarius succinctus and Nassarius variciferus) to reveal their genetic patterns of TTX accumulation and resistance. For genetic expression, the cellular and metabolic process, and binding and catalytic activity accounted for the top classification categories for both species. The toxic communities generally produced more up-regulated genes than non-toxic communities. The mostly different expression genes among toxic and non-toxic communities mainly included heat shock protein 83-like, cytochrome c oxidase subunit, WAS protein family member 2, delta-aminolevulinic acid dehydratase, protein transport protein Sec24D isoform X2 and so on, some of which referred to detoxification. In mutation level, the sodium channel gene of N. succinctus had one amino acid "L" that is different from that of other animals. The transcriptome analysis of both toxic and nontoxic communities in two Nassarius species provided important genetic basis for adaptive evolution research of TTX accumulation and resistance.
\end{abstract}

\section{INTRODUCTION}

As potent neurotoxin, Tetrodotoxin (TTX) and Saxitoxin (STX) can specifically bind to sodium channels of animals by sharing one binding site with high affinities ${ }^{4,5,6}$, which could lead to poisoning and death of animals. However, some animals can accumulate TTX or STX in their body to defense the external environment since they possibly could be resistant to toxins by target site insensitivity, such as in fish, frog and mullusca ${ }^{1,2,3,32}$. Understanding how TTX is accumulated in animals and how animals resist to TTX in genetic level would be particularly critical for adaptive evolution research and toxin poisoning cure in medicine.

Some proteins which were possibly related with TTX binding and transportation were found in puffer fish ${ }^{2,17}$. Takashi et al $(2000)^{34}$ firstly purified some TTX-binding proteins in puffer fish. Yotsu-Yamashita et al (2010 and 2013) $)^{2,17}$ reported the distribution and localization of TTX-binding protein and their homologous proteins in puffer fish, which possibly be related with TTX transportation. Feroudj et al (2014) ${ }^{19}$ employed DNA microarray analysis revealed some gene candidates related with TTX accumulation in puffer fish ${ }^{19}$. But the detailed accumulation mechanism of TTX is needed to be well understood in a wide range of animals. Except pufferfish, STX and TTX resistance also appears in various kinds of animals, such as snails ${ }^{7}$, xanthid $\mathrm{crab}^{8}$, blue-ringed octopus ${ }^{9}$, and and frogs ${ }^{3,10-12}$. It has been reported that the mutation in sodium channel genes $^{13}$ can block the binding of toxin and thus result in the toxin resistance ${ }^{1,2,3,14-16}$. Understanding how organisms resist TTX on genetic level would contribute to investigate adaptive evolution in ecological communities.

Nassarius, a species-rich genus of Nassariinae, is important in maintaining the balance of marine ecological system. For a long time, Nassarius is consumed as popular food in Asian countries as economic species ${ }^{21}$. 
However, eating nassariids of Nassarius have caused hundreds of food poisoning and death incidents in the last several years, especially in China. Our previous studies detected that the food poisoning incident was due to TTX accumulated in Nassarius sp's body and the toxicity of different Nassarius species and different populations within oneNassarius species was different ${ }^{22}$, which means that some Nassarius species and populations can accumulate TTX in their body but some others do not accumulate. This is possibly related with the sea environments they live which could lead to adaptive evolution for different communities.

Currently the genetic basis involving TTX accumulation and resistance inNassarius is unclear. Here we performed transcriptional analysis for both toxic and non-toxic specimens in two Nassarius species (Nassarius succinctus and Nassarius variciferus), aiming to reveal their genetic patterns for TTX accumulation and resistance in genetic expression and mutation level for better understanding the adaptive evolution for toxin.

\section{MATERIALS AND METHODS}

Sample collection, treatment and toxicity test. The samples used in this study have been tested for the TTX toxicity in our previous published research ${ }^{22}$. Based on the toxicity test, $N$. succinctus and $N$. variciferus were selected for transcriptional analysis since they included both toxic and non-toxic specimens. The liver and pancreas were treated for RNA extraction and sequencing since they contained more toxin. For RNA-seq protocol, the toxic and non-toxic specimens were sequenced as treatment group and control group respectively for each species. We selected three toxic specimens and three non-toxic specimens as triple repeat for treatment group and control group respectively, for each of $N$. succinctusand N. variciferus . The detailed sample collection was shown in Fig. 1.

RNA library preparation, transcriptome and gene assembly. After RNA extraction the mRNA were fragmented with fragmentation buffer and were used to synthesize cDNA. Short cDNA fragments were purified and then resolved with end reparation and adapter connection, from which fragments with suitable length were selected for PCR enrichment. Agilent 2100 Bioanaylzer and ABI StepOnePlus Real-Time PCR System was used for qualification. Finally, the library was sequenced using Illumina HiSeq $4000^{23}$.

The Trinity (v2.0.6) ${ }^{24}$ was used to perform de novo assembly after filtering out raw reads. The assembly quality was evaluated with N50 and ortholog hit ratios (OHR). Paired reads passing the filter were then concatenated using Concatenate datasets (version 1.0.0) in both the right and left direction. Transcript abundancies were calculated by RSEM version 1.1.1754 ${ }^{25-26}$ using the pool of non-normalized reads with default settings.

Differentially expressed gene analysis. The functional annotation was performed from databases of NT, NR, GO, COG, KEGG, SwissProt and InterPro. With BLASTX the final assembly was submitted to these databases with the threshold of E-value [?] 10-5 ${ }^{27}$. The NR and InterProScan5 were used to get $\mathrm{GO}$ annotation and InterPro annotation respectively. Unigenes were obtained from mapping clean reads by Bowtie $2^{28}$. The gene expression level was calculated with RSEM. The PCA analysis was performed with all samples by princomp, a function of R. We detected DEGs (Differentially Expressed Genes) with Possion Dis as requested ${ }^{29}$.

Q-PCR confirmation. Real-time PCR was performed with the SG Fast qPCR Master Mix (High Rox) using the cDNA synthetized above. Cycle threshold values were normalized to the gene 18S. A total of 18 DEGs closely related with coding toxin-binding protein were selected as target Q-PCR functional genes. Fold changes were determined with the relative expression software tool.

Mutations in sodium channels genes. Based on the transcript unigenes of all samples, we retrieved the $\mathbf{s}$ odium channels genes to which the TTX binds. We downloaded available $\mathbf{s}$ odium channels genes from mollusca, human, mouse and others in NCBI as reference database. Then all unigenes of $N$. succinctus and $N$. variciferus were mapped to the reference database. While the unigenes were best matched to the reference sequences specific primers were designed to sequence them for confirmation.

\section{RESULTS}


Quality of sequencing data and assembly. A total of 12 samples were used for transcriptome sequencing in this study, half out of which were from $N$. succinctus and half out of which were from $N$. variciferus. For each of $N$. succinctus and $N$. variciferus , 3 specimens (triple repeat) were toxic as treat group and 3 specimens (triple repeat) were non-toxic as control group. A total of $39.96 \mathrm{~Gb}$ bases and $40.19 \mathrm{~Gb}$ bases were generated for all specimens of $N$. succinctusand all specimens of $N$. variciferus respectively based on Illumina Hiseq sequencing. The clean reads quality metrics after filtering sequences containing low-quality, adaptor-polluted and high content of unknown base $(\mathrm{N})$ reads were shown in Table 1 . The distribution of base content and quality were shown in Figure S1. These reads quality indicated that the transcriptome sequencing performed well for biological functional analysis. Accession numbers of 12 samples in Genbank were: SRR10582953, SRR10582959, SRR10582955, SRR10582954, SRR10582956, SRR10582961, SRR10582957, SRR10582958, SRR10582960, SRR10582963, SRR10582963, SRR10582964.

Gene functional annotation. For both $N$. succinctus and $N$. variciferus, the $\mathrm{Nr}$ and $\mathrm{Nt}$ databases got higher annotation proportion than other databases, with more than $30 \%$ (Table S1). The overall annotation proportion for $N$. succinctus and $N$. variciferus was $52.61 \%$ and $55.07 \%$ respectively. The distribution of annotated species with NR was shown in Fig. 2. The annotated species were generally consistent between $N$. succinctus and N. variciferus, including Aplysia californica, Oncorhynchus mykiss, Octopus bimaculoides , Monosiga brevicollis MX1, Lottia gigantea , Biomphalaria glabrata , Crassostrea gigas , Trichuris suis , Exaiptasia pallida, Lingula anatine and Mus musculus. Among these species annotated,A. californica showed the highest annotation proportion. The coding sequence (CDS) was selected from segment of unigenes that best mapped to functional databases. For some unigenes which were not annotated, the ESTScan was used to predict the $\mathrm{CDS}^{30}$. For both $N$. succinctus and $N$. variciferus, the total number of CDS was between 10000-80000 and the mean length was 300-500bp. There was no much difference for the CDS prediction between the two species.

Gene expression patterns and different expressed genes. After assembly and mapping clean reads to unigenes, the gene expression level for each sample was calculated by PCA analysis. As shown in Fig. 3, the expression level between the toxic and non-toxic specimens were generally different. The non-toxic $N$. succinctus specimens from Dalian showed more similar gene expression pattern. Two toxic specimens of $N$. variciferus from Lianyungang had different expression from the non-toxic samples from Dalian.

The different expressed genes (DEGs) revealed up-regulated and down-regulated genes between toxic and non-toxic specimens for both species (Fig. 4). Compared with the non-toxic specimens (ZD) in N. variciferus , each toxic specimens (ZL) showed more up-regulated genes. For N. succinctus, the toxic specimens (HL) also produced more up-regulated genes in comparison with the non-toxic specimens (HQ). The Gene Ontology (GO) classification and functional enrichment was performed for DEGs, including three ontologies of molecular function, cellular component and biological process. N. variciferus and $N$. succinctus produced coincident patterns of functional enrichment, for which the cellular and metabolic process from biological process, and binding and catalytic activity from molecular function accounted for the top classification categories (Figure S2).

The most common different expressed genes were obtained from toxic (treatment) and non-toxic (control) groups for both species (Table 2). Compared with the non-toxic groups, the most upregulated genes from toxic groups included heat shock protein 83-like, cytochrome c oxidase subunit I and II, protein transport protein Sec24D isoform X2, WAS protein family member 2, delta-aminolevulinic acid dehydratase, and others. The pathways related with the main upregulated genes were protein processing in endoplasmic reticulum, Oxidative phosphorylation, Metabolic pathways, Cardiac muscle contraction, Thermogenesis, Non-alcoholic fatty liver disease (NAFLD), Alzheimer disease, Parkinson disease, Huntington disease, Porphyrin and chlorophyll metabolism Metabolic pathways, and so on. On the other hand, the pathways related with the downregulated genes were mainly about transcription, like spliceosome, RNA transport, basal transcription factors.

For accuracy confirmation of transcriptome sequencing, multiple DEGs found as potential functional genes were selected for $\mathrm{qPCR}$. The $\mathrm{qPCR}$ of these selected genes was performed for both toxic specimens (treat 
group) and non-toxic specimens (control group). The $18 \mathrm{~S}$ was selected as the reference gene. In general, the statistic results indicated that the $2-([?][?] \mathrm{Ct})$ and actual expression level for treat and control groups showed consistent tendency as in the Fig. 5.

Sodium channel genes. Based on the transcript unigenes of both species, we tried to retrieve the sodium channel genes for both species. A total of 3425bp sequence (unigene CL8899.Contig1_All) from N. succinctus was best matched to the sodium channels genes in reference database with identify score with more than $80 \%$. The amino acids of this matched unigene was identified as Domain II and Domain III of sodium channels. Among the amino acid sites, we found one new amino acid ' $\mathrm{L}$ ' in Domain II, in comparison with all other species (Fig. 6). By PCR confirmation, we obtained the same sequence of this new found sodium channels gene from $N$. succinctus. Unfortunately, no perfect matched sodium channels genes were found from unigenes of $N$. variciferus even though we blasted the clean reads of both species (150bp) to all reference sequences.

\section{DISCUSSION}

More and more researches pointed that animals could accumulate toxin, like PSP, TSX and TTX in their body and thus produce toxin resistance ${ }^{31,32}$. It is significant to reveal the genetic basis of toxin accumulation and resistance in various of animals accumulating toxin, not only for adaptive evolution understanding but also for food-poisoning cure in medicine. It has been reported that the toxin resistance could be due to the mutation of sodium channel genes in animals where the toxin specially bind to ${ }^{1,13,31-, 32}$. But the accumulation mechanism of TTX in animals was uncertain. Different environments can lead to the adaptive evolution of animals with TTX. Species in Nassarius have toxic and non-toxic communities from different sea areas. In this study, as typical TTX-resistant species, $N$. succinctus and $N$. variciferuswere used to reveal their genetic expression and mutation patterns from toxic and non-toxic communities.

First of all, N. succinctus and N. variciferusdemonstrated similar DEG patterns. Generally the toxic communities produced more up-regulated genes than the non-toxic communities. For $N$. variciferus, the upregulated genes of toxic specimens was significantly more than that of non-toxic specimens. For $N$. succinctus , two toxic specimens showed more up-regulated genes. This may suggest that special mechanism activities associated with TTX accumulation and resistance occur in toxic samples. The PCA analysis of DEGs indicated that the gene expression patterns of toxic specimens was different from that of non-toxic specimens for both species. Furthermore, we selected the most common DEGs (both upregulated and downregulated genes) between the toxic and non-toxic groups for both species. Compared with the non-toxic groups, the heat shock protein and cytochrome c oxidase subunit were the most significant upregulated genes from toxic groups. The heat shock protein involved the pathway of protein processing in endoplasmic reticulum. One of the most important functions of endoplasmic reticulum is detoxification, which is the removal of all the toxic materials such as metabolic wastes or drugs ${ }^{35-36}$. This strongly suggest that the TTX inNassarius 's body is possibly removed by special mechanism. Cytochrome c oxidase subunit I and II involve the pathways of various diseases like Oxidative phosphorylation, Metabolic pathways, Cardiac muscle contraction, Non-alcoholic fatty liver disease (NAFLD), Alzheimer disease, Parkinson disease and Huntington disease. The cytochrome c oxidase subunit plays key role in respiration. Thus, it is reasonable that the cytochrome c oxidase subunit is highly upregulated in specimens accumulating TTX.

All the transcriptome unigenes were clustered against the sodium channel gene references to get the sodium channel genes of Nassarius. We obtained D2 and D3 domains of N. succinctus with length of 3425bp where one amino acid site "L" was different from that of all other animals possessing TTX resistance. Whether this new amino acid "L" is a potential mutational site with TTX resistance should be further confirmed by electrophysiology. Unfortunately, the D1 and D4 domains of Nassarius could not be obtained by unigene blast, reads blast or RACE-PCR. We suggest the reasons may be that the RNA-seq sequences do not cover the sodium channel regions due to RNA degradation or that the sodium channel genes of Nassarius are complicated for assembly with RNA-seq. In the future studies, we would employ comprehensive samples and more advanced sequence technologies to explore the complete sodium channel genes of various toxic and non-toxic communities in different Nassarius species. 


\section{Conclusion}

This study revealed the genetic patterns of TTX accumulation and resistance in two Nassarius species by transcriptome analysis. For gene expression, the cellular and metabolic process and binding and catalytic activity accounted for the top classification categories for both $N$. succinctus and $N$. variciferus. The toxic specimens showed more upregulated genes compared with the non-toxic specimens bothN. succinctus and $N$. variciferus . The heat shock protein and cytochrome c oxidase subunit were found as the most significant different expression genes between all the toxic and non-toxic groups, which are mainly related with the metabolic mechanism of detoxification and various diseases. Compared with other animals possessing toxin resistance, one new amino acid " $\mathrm{L}$ " was found in the sodium channels of $N$. variciferus, which could possibly be the mutation for blocking the TTX-binding. Future studies should focus on exploring the complete sodium channel genes of various Nassarius species and detailed metabolic pathways for TTX resistance in Nassarius .

\section{Acknowledgments}

This study was supported by a grant from "The National Natural Science Foundation of China (31600294)" and China Scholarship Council. We also thank the support by the Bioinformatics Center of Nanjing Agricultural University, and Biological Computer Platforms of UC Berkeley, CA.

\section{Reference:}

1.Venkatesh, B., Lu, S.Q., Dandona, N., See, S.L., Brenner, S. et al. Genetic basis of tetrodotoxin resistance in pufferfishes. Current Biology $15,2069-2072$ (2005)

2.Yotsu-Yamashita, M., Yamaki, H., Okoshi, N. \& Araki, N. Distribution of homologous proteins to puffer fish saxitoxin and tetrodotoxin binding protein in the plasma of puffer fish and among the tissues of fugu pardalis examined by western blot analysis. Toxicon55 ,1119-1124 (2010)

3.Tarvin, R.D., Borghese, C.M., Sachs, W., Santos, J.C., Lu, Y. et al. Interacting amino acid replacements allow poison frogs to evolve epibatidine resistance. Science $357,1261-1266$ (2017)

4.Narahashi, T., Moore, J.W. \& Poston, R.N. Tetrodotoxin derivatives:chemical structure and blockage of nerve membrane conductance. Science $156,976-979$ (1967)

5. Kao, C.Y. Actions of nortetrodotoxin on frog muscle and squid axon. Toxicon Official Journal of the International Society on Toxinology $20,1043-1050$ (1982)

6. Soong, T.W. \& Venkatesh, B. Adaptive evolution of tetrodotoxin resistance in animals. Trends in Genetics $22,621-626(2006)$

7. Narita, H., Noguchi, T., Maruyama, J., Ueda, Y., Hashimoto, K. et al. Occurrence of tetrodotoxin in a trumpet shell, "boshubora" Charonia sauliae. Bull. Japanese. Soc. Sci. Fish 47 ,935-94 (1981)

8. Noguchi, T., Uzu, A., Koyama, K., Maruyama, J. et al. Occurrence of tetrodotoxin as the major toxin in a xanthid crab, Atergatis floridus. Bull. Japanese. Soc. Sci. Fish 49 ,1887-189 (1983)

9. Sheumack, D.D., Howden, M.E.H. \& Spence, I. Occurrence of a tetrodotoxin-like compound in the eggs of the venomous blue-ringed octopus (hapalochlaena maculosa). Toxicon Official Journal of the International Society on Toxinology $22,811-81(1984)$

10. Mosher, H.S., Fuhrman, F.A., Buchwald, H.D. \& Fischer, H.G. Tarichatoxin-tetrodotoxin: a potent neurotoxin. Science44,1100-1110 (1964)

11. Noguchi, T. \& Hashimoto, Y. Isolation of tetrodotoxin from a goby gobius criniger. Toxicon Official Journal of the International Society on Toxinology $11,305-307$ (1973)

12. Kim, Y., Brown, G. \& Mosher, F. Tetrodotoxin: occurrence in atelopid frogs of costa rica. Science $\mathbf{1 8 9}$ ,151-152 (1975) 
13. Mcglothlin, J.W., Chuckalovcak, J.P., Janes, D.E., Edwards, S.V., Feldman, C.R. et al. Parallel evolution of tetrodotoxin resistance in three voltage-gated sodium channel genes in the garter snake thamnophis sirtalis. Molecular Biology and Evolution .11 (2014)

14. Geffeney, S.L., Fujimoto, E., Brodie, E.D., Brodie, E.D. \& Ruben, P.C. Evolutionary diversification of ttx-resistant sodium channels in a predator-prey interaction. Nature $434,759-763$ (2005)

15. Jost, M.C., Hillis, D.M., Lu, Y., Kyle, J.W., Fozzard, H.A. \& Zakon, H.H. Toxin-resistant sodium channels: parallel adaptive evolution across a complete gene family. Molecular Biology and Evolution25 ,1016-1024 (2008)

16. Hanifin, C.T. \& Gilly, W.F. Evolutionary history of a complex adaptation: tetrodotoxin resistance in salamanders. Evolution69 232-244 (2015)

17. Yotsu-Yamashita, M., Okoshi, N., Watanabe, K., Araki, N., Yamaki, H. et al. Localization of pufferfish saxitoxin and tetrodotoxin binding protein (pstbp) in the tissues of the pufferfish, takifugu pardalis, analyzed by immunohistochemical staining. Toxicon $\mathbf{7 2}, 23-28$ (2013)

18. Yotsu-Yamashita, M., Nishimori, K., Nitanai, Y., Isemura, M., Sugimoto, A. Binding properties of 3h-pbtx-3 and 3h-saxitoxin to brain membranes and to skeletal muscle membranes of puffer fish fugu pardalis and the primary structure of a voltage-gated na+ channel $\alpha$-subunit (fmna1) from skeletal muscle off. pardalis. Biochemical \& Biophysical Research Communications $267,403-412$ (2000)

19. Feroudj, H., Matsumoto, T., Kurosu, Y., Kaneko ,G., Watabe, S. et al. Dna microarray analysis on gene candidates possibly related to tetrodotoxin accumulation in pufferfish. Toxicon 77 ,68-72 (2013)

20. Casewell, N.R., Wagstaff, S.C., Harrison, R.A., Camila, R., Renjifo, C. et al. Domain loss facilitates accelerated evolution and neofunctionalization of duplicate snake venom metalloproteinase toxin genes. Molecular Biology and Evolution 28 , 2637-2649 (2011)

21. Zou, S., Li, Q. \& Kong, L. Monophyly, distance and character-based multigene barcoding reveal extraordinary cryptic diversity in nassarius: a complex and dangerous community. Plos One 7 ,e47276 (2012)

22. Zou, S., Song, J., Wang, C. \& Wang, C. The relationships between toxicity, species and populations in nassarius based on toxin detection and multiple gene barcoding. Journal of Ocean University of China18 , 1515-1522 (2019)

23. Conesa, A., Madrigal, P., Tarazona, S., Gomez-Cabrero, D., Cervera, A., Mcpherson, A. et al. A survey of best practices for rna-seq data analysis. Genome Biology 17, 181(2016)

24. Grabherr, M.G., Haas, B.J., Yassour, M., Levin, J.Z., Thompson, D.A. et al. Full-length transcriptome assembly from rna-seq data without a reference genome. Nature Biotechnology 29 ,644-652 (2011)

25. Haas, B.J., Papanicolaou, A., Yassour, M., Grabherr, M., Blood, P.D. et al. De novo transcript sequence reconstruction from rna-seq using the trinity platform for reference generation and analysis. Nature Protocols 8 ,1494-1512 (2013)

26. Dewey, C.N. \& Bo, L. Rsem: accurate transcript quantification from rna-seq data with or without a reference genome. BMC Bioinformatics12,323-323 (2011)

27. Conesa, A., Gotz, S., Garcia-Gomez, J.M., Terol, J., Talon, M. et al. Blast2go: a universal tool for annotation, visualization and analysis in functional genomics research. Bioinformatics21,3674-3676 (2005)

28. Langmead, B. \& Salzberg, S.L. Fast gapped-read alignment with bowtie 2. Nature Methods. 9 ,357-359 (2012)

29. Audic, S. \& Claverie, J.M. The significance of digital gene expression profiles. Genome Res 7 , 986-995 (1997) 
30. Iseli, C., Jongeneel, C.V. \& Bucher, P. Estscan: a program for detecting, evaluating, and reconstructing potential coding regions in est sequences. Proceedings. International Conference on Intelligent Systems for Molecular Biology 138-48 (1999)

31. Bricelj, V.M., Connell, L., Konoki, K., Macquarrie, S.P., Scheuer, T. et al. Sodium channel mutation leading to saxitoxin resistance in clams increases risk of psp. Nature $434,763-767$ (2005)

32. Li, Y., Sun, X., Hu, X., Xun, X., Zhang, J. et al. Scallop genome reveals molecular adaptations to semi-sessile life and neurotoxins. Nature Communications. 8 ,1721 (2017)

33. Lee, J.H., Kondo, H., Sato, S., Akimoto, S., Saito, T. et al. Identification of novel genes related to tetrodotoxin intoxication in pufferfish. Toxicon $49,939-953$ (2007)

34. Takashi, M., Kunio, Y., Kiyoshi, F., Michiko, K. Purification and some properties of a tetrodotoxin binding protein from the blood plasma of kusafugu, Takifugu niphobles. Toxicon. 38 , $463 \pm 468$ (2000).

35. Fabienne, F., Bernard, F. Role of endoplasmic reticulum stress in drug-induced toxicity. Pharmacology Research \& Perspectives.4 (1),e00211 (2016).

36. Sprooten J., Garg, A.D. Type I interferons and endoplasmic reticulum stress in health and disease. International review of cell and molecular biology, 350 ,63-118 (2020).

\section{Figure legends}

FIGURE 1 Toxic and non-toxic communities of $N$. succinctus and $N$. variciferus collected from China coast.

FIGURE 2 Distribution of annotated species for both $N$. succinctus and $N$. variciferus which show similar annotated patterns. The Aplysia california species take accounted for the top proportion.

FIGURE 3 PCA plot for difference of expression level between the toxic and non-toxic communities (ZD \& HD: non-toxic specimens, ZL \& HL: toxic specimens). It indicated that the toxic and non-toxic communities generally show similar expression patterns.

FIGURE 4 DEGs patterns between toxic and non-toxic specimens of $N$. succinctus and $N$. variciferus. HD inN.succinctus and ZD in N.variciferus (non-toxic samples) were set as control group and HL and ZL were set as treatment group. Examples: HD1-VS-HL3 means that the HL3 generated more up-regulated genes (blue) than down-regulated (orange) in comparison with HD1. ZD1-VS-ZL3 means that the ZL3 generated more up-regulated genes (orange) than down-regulated (blue) in comparison with ZD1.

FIGURE 5 Consistency of 2 -([?][?]Ct) and actual expression level by RT-PCR. Six potential DEGs related with TTX accumulation and resistance was performed.

FIGURE 6 Potential amino acids mutation site related with TTX resistance on sodium channel of $N$. succinctus. The site indicated in yellow is newly identified by our study.

FIGURE S1 Distribution of base content and quality for all specimens studied.

FIGURE S2 The top classification categories for the DEGs of both species.

TABLE S1 Functional annotation for both Nassariusspecies from 7 databases

Table 1 . The clean reads quality metrics after Quality control.

\begin{tabular}{llllll}
\hline Sample & Total Raw Reads $(\mathrm{Mb})$ & Total Clean Reads $(\mathrm{Mb})$ & Total Clean Bases $(\mathrm{Gb})$ & Clean Reads Q20(\%) & Clean Reads \\
\hline HL1 & 58.37 & 44.66 & 6.7 & 98.09 & 94.47 \\
HL2 & 59.9 & 44.32 & 6.65 & 97.88 & 93.98 \\
HL3 & 55 & 44.12 & 6.62 & 98.27 & 94.7 \\
HD1 & 60.43 & 44.31 & 6.65 & 98.19 & 94.59
\end{tabular}




\begin{tabular}{llllll}
\hline Sample & Total Raw Reads $(\mathrm{Mb})$ & Total Clean Reads $(\mathrm{Mb})$ & Total Clean Bases $(\mathrm{Gb})$ & Clean Reads Q20(\%) & Clean Reads \\
\hline HD2 & 60.55 & 44.24 & 6.64 & 98.42 & 95.42 \\
HD3 & 62.14 & 44.67 & 6.7 & 98.45 & 95.53 \\
ZL1 & 63.16 & 44.59 & 6.69 & 96.85 & 90.95 \\
ZL2 & 63.16 & 44.47 & 6.67 & 96.75 & 90.71 \\
ZL3 & 63.16 & 44.86 & 6.73 & 96.74 & 90.65 \\
ZD1 & 59.92 & 44.65 & 6.7 & 96.88 & 91.04 \\
ZD2 & 59.92 & 44.91 & 6.66 & 96.86 & 91.05 \\
ZD3 & 61.54 & 44.41 & & 96.85 & 91.03 \\
\hline
\end{tabular}

Table 2 Most different expressed genes among all toxic (treatment) and non-toxic (control) groups of two species

\begin{tabular}{|c|c|c|c|}
\hline Definition & Expression trend & Length & Pathway \\
\hline heat shock protein 83-like & up & 1906 & Protein processing il \\
\hline cytochrome c oxidase subunit II & up & 673 & Oxidative phosphory \\
\hline cytochrome c oxidase subunit I & up & 1855 & Oxidative phosphory \\
\hline histone H1E-like & up & 686 & NA \\
\hline secreted frizzled-related protein 5 & up & $597-737$ & Wnt signaling pathy \\
\hline WAS protein family member 2 & up & $436-611$ & Adherens junction; I \\
\hline serine-rich adhesin for platelets-like isoform X1 & up & $3044-3563$ & NA \\
\hline mucin-2 & up & $1507-1399$ & Amoebiasis Gastric \\
\hline ATP-dependent RNA helicase A & up & $415-678$ & NA \\
\hline DEAH (Asp-Glu-Ala-His) box polypeptide 9 & up & $382-852$ & NA \\
\hline keratin, type II cytoskeletal 1-like & up & 453 & NA \\
\hline ATP-dependent RNA helicase A-like & up & 953 & NA \\
\hline uncharacterized protein LOC102093901 & up & 1369 & NA \\
\hline heterogeneous nuclear ribonucleoprotein A2/B1 & up & 570 & NA \\
\hline delta-aminolevulinic acid dehydratase & up & 2207 & Porphyrin and chlor \\
\hline signal recognition protein & up & $2221-3222$ & Protein export \\
\hline aminolevulinate, delta-, dehydratase & up & 986 & Porphyrin and chlor \\
\hline GH24401 gene product from transcript GH24401-RA & up & 465 & NA \\
\hline heterogeneous nuclear ribonucleoprotein A1b & up & 376 & Spliceosome \\
\hline human immunodeficiency virus type I enhancer binding protein 1 & up & 263 & NA \\
\hline protein transport protein Sec24D isoform X2 & up & 1124 & Protein processing i \\
\hline LOW QUALITY PROTEIN: RNA-binding protein 25 & up & 979 & Spliceosome \\
\hline tissue factor pathway inhibitor & up & 861-1061 & Complement and co \\
\hline AGAP011395-PA & up & 823 & Hedgehog signaling \\
\hline mucin-5B & down & $1533-1556$ & IL-17 signaling path \\
\hline uncharacterized protein & down & 1315 & NA \\
\hline myosin light polypeptide 6 -like & down & 738 & NA \\
\hline myosin heavy chain, striated muscle isoform $\mathrm{X} 1$ & down & $4214-7132$ & NA \\
\hline oviductin [EC:3.4.21.120] & down & 1079 & NA \\
\hline protocadherin-15b precursor & down & 632 & NA \\
\hline pre-mRNA-processing factor 40 homolog B & down & 736 & Spliceosome \\
\hline troponin $\mathrm{T}$ isoform $\mathrm{X} 1$ & down & 2844 & NA \\
\hline histone-lysine N-methyltransferase $2 \mathrm{C}$ & down & 1826 & Lysine degradation \\
\hline low-density lipoprotein receptor-related protein 4 -like & down & 751 & NA \\
\hline ligand-gated ion channel 4-like & down & 484 & NA \\
\hline mucin-13 & down & 1028 & NA \\
\hline
\end{tabular}




\begin{tabular}{llll}
\hline Definition & Expression trend & Length & Pathway \\
\hline nuclear pore complex protein Nup205 & down & 548 & RNA transport \\
C-type lectin domain family 4 member M-like & down & 780 & NA \\
TATA-box binding protein associated factor 3 L homeolog & down & 751 & Basal transcription f \\
tektin-3 & down & 3356 & NA \\
mucin 2, oligomeric mucus/gel-forming & down & 1396 & NA \\
very low density lipoprotein receptor & down & 1456 & Spinocerebellar atax \\
ncharacterized protein & down & 255 & NA \\
N-acetylated-alpha-linked acidic dipeptidase [EC:3.4.17.21] & down & 1035 & NA \\
\hline
\end{tabular}

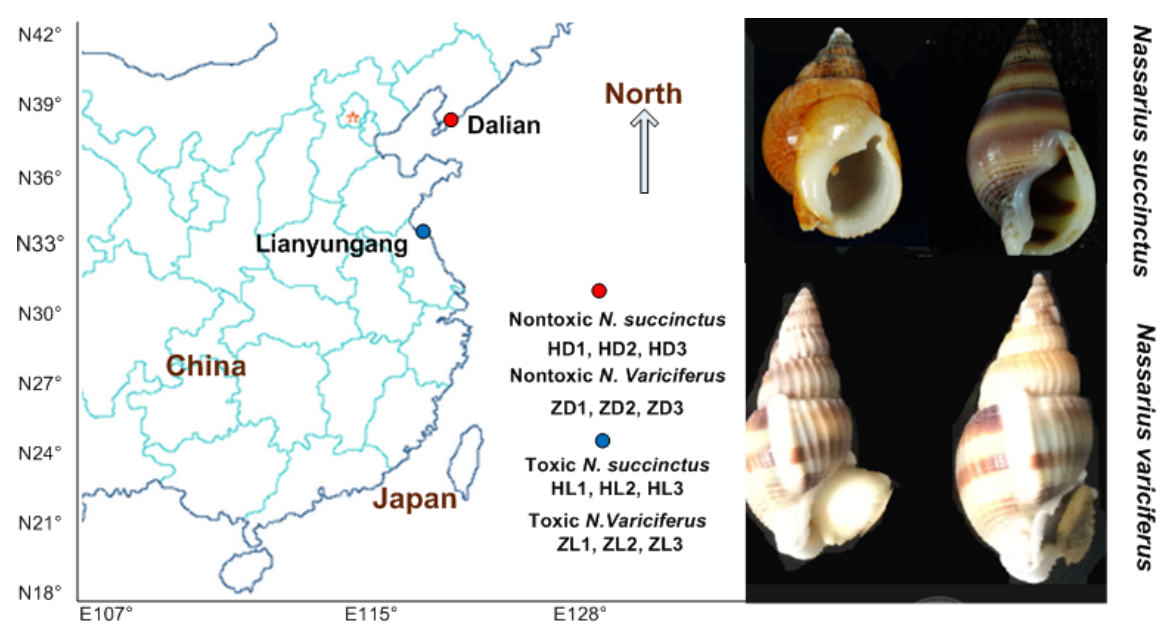

Fig. 1

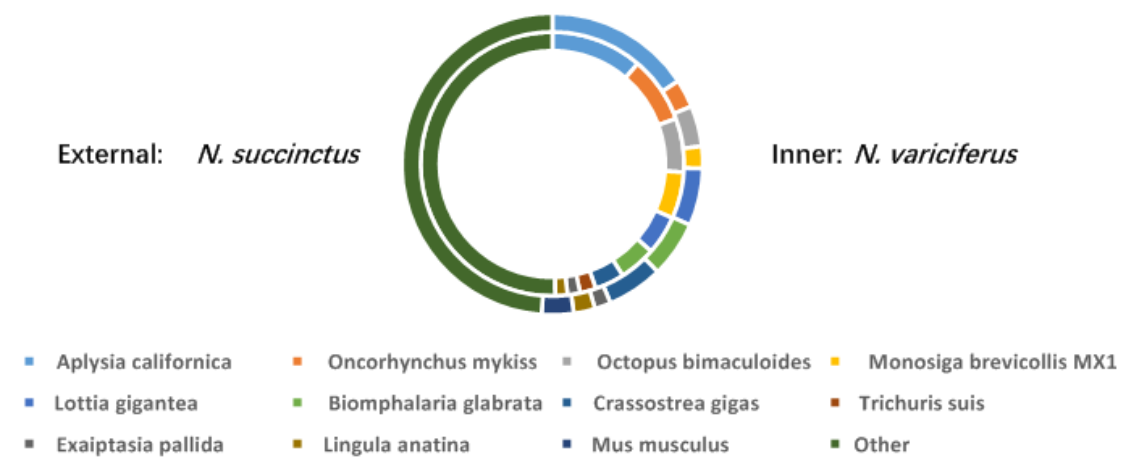

Fig. 2 

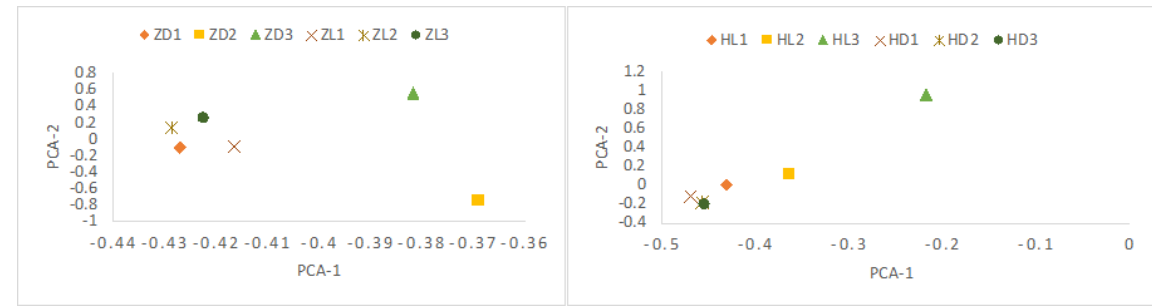

Fig. 3

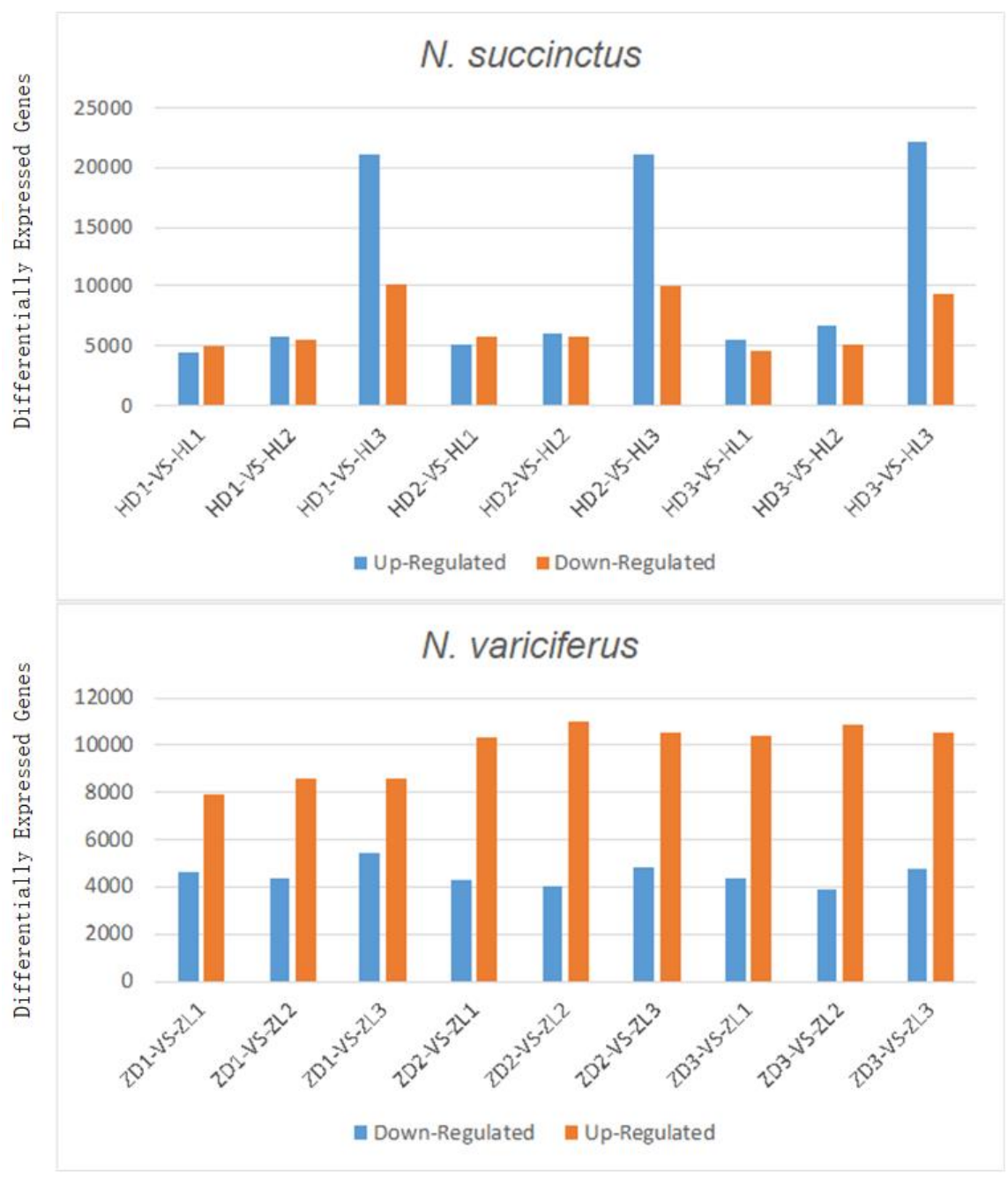

Fig. 4 


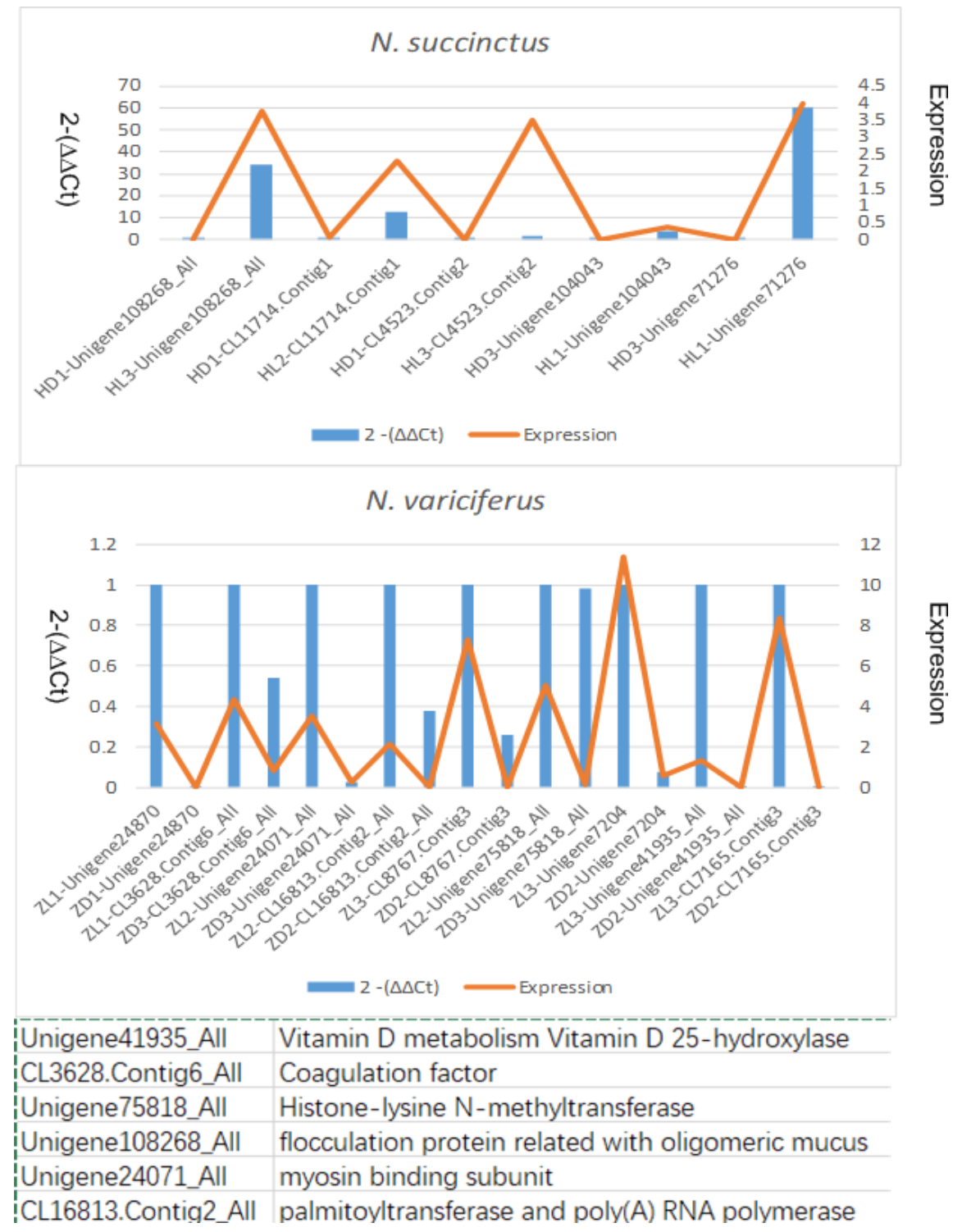

Fig. 5 
Species name

Rattus norvegicus

Takifugu pardalis

Drosophila melanogaster

Loligo opalescens

Aplysia california

Bdelloura candisda

Cyanea caplillata

Mya areneria sensitive

Mya areneria resistant

Homo sapidens

Tetraonon nigroviridis-a

Tetraonon nigroviridis- $b$

Takifugu rubripes

Chlamys farreri

Crassostrea gigas

Solemya velum

Patinopecten yessoensis

N. succinctus (TTX-resistance)
Domain II

R V L C GE WI E T VATFK G WMD I

R I L C GE WI ET I A T F K GWMD I

$R \vee L C G E W I E S \quad V A T F K G W I Q I$

$R V L C G E W I E S \quad V A T F K G W I N$ I

$R \vee L C G E W I E S \quad V A T Y K G W I D I$

$R \vee L C G E Y I E S \quad V A T F K G W T D$ I

RI LCGKWIEP TATLE GWFE

$R \vee L C G E W I E S \quad V A T Y K G W I D I$

RVLCGEWI DS VATYKGWI DI

R I L C GE WI ET VATFK GWMDI

$R$ | LCGE WIEN | ATFKG WTA I

$R \vee L C G E W I D T V A T F K G W M E$ I

RVLCGE WI ES VATFKGWTDI

$R V L C G E W I E S \quad V A T Y K G W T V I$

$R V L C G E W I$ QS VATY GWI EV

RVLCGEWI QS VATYKGWI E I

R V L C GEWI ES VATYKGWT VI $R \vee L C G E W I E L \quad V A T F E G W I E I$

Fig. 6
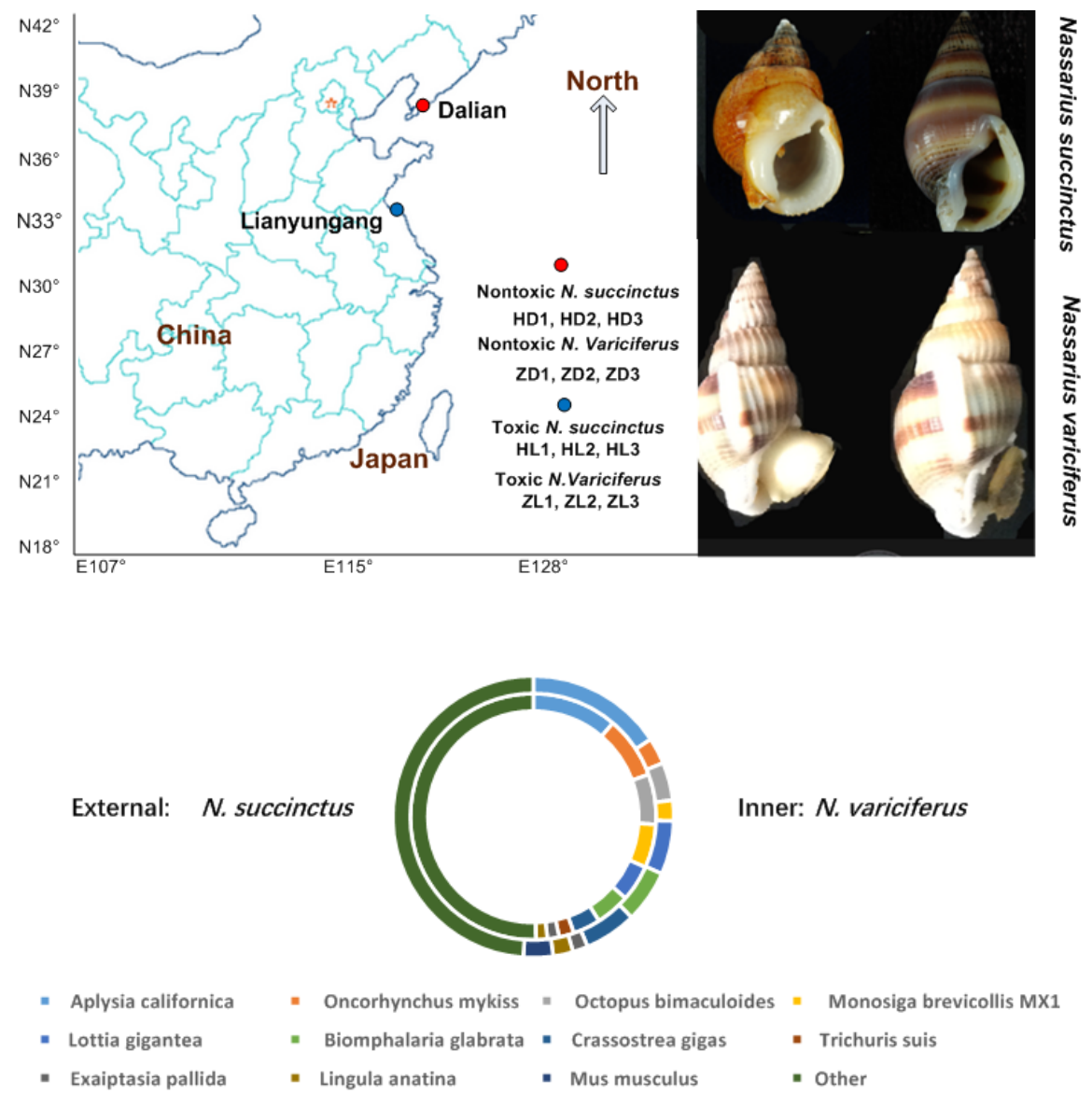

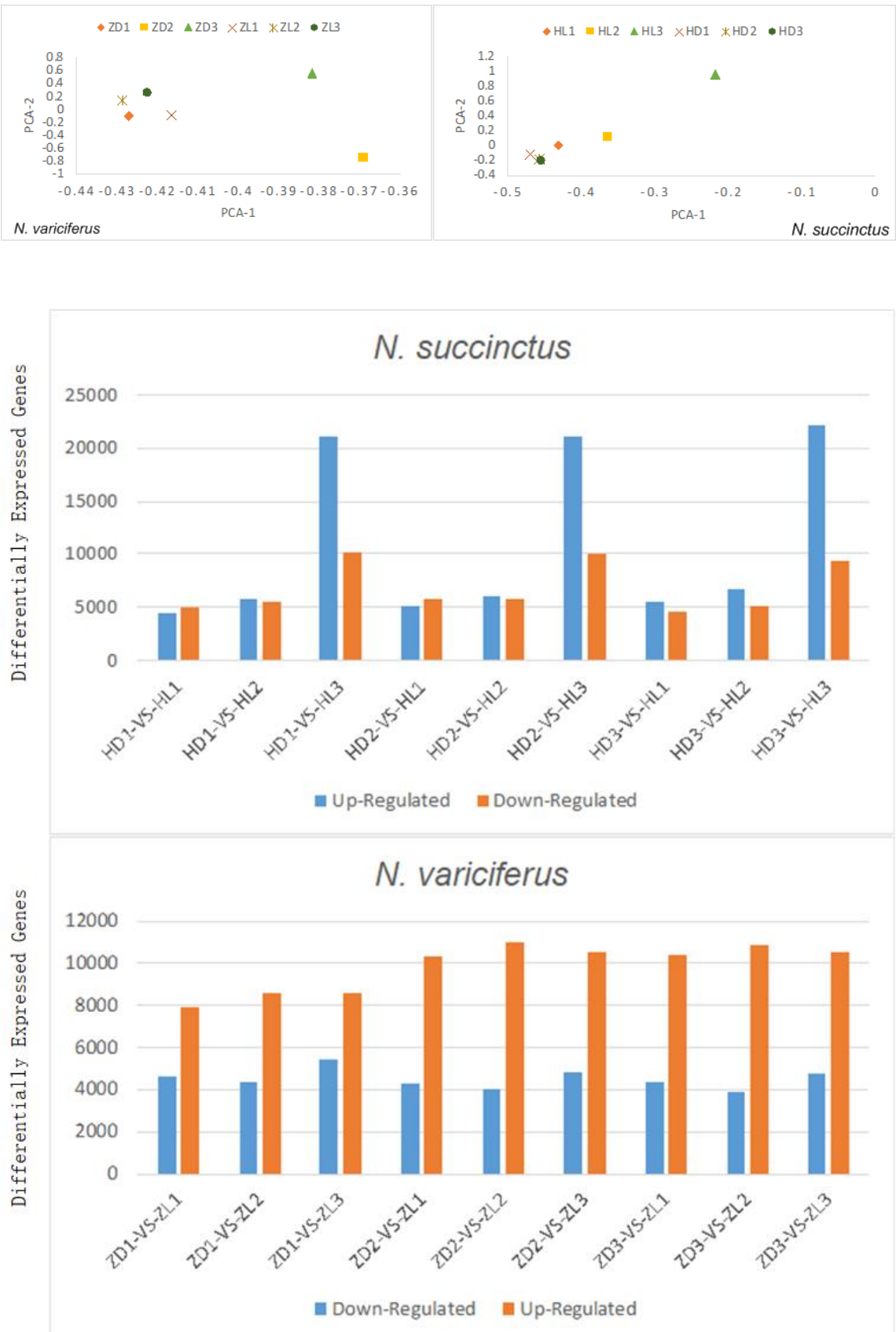


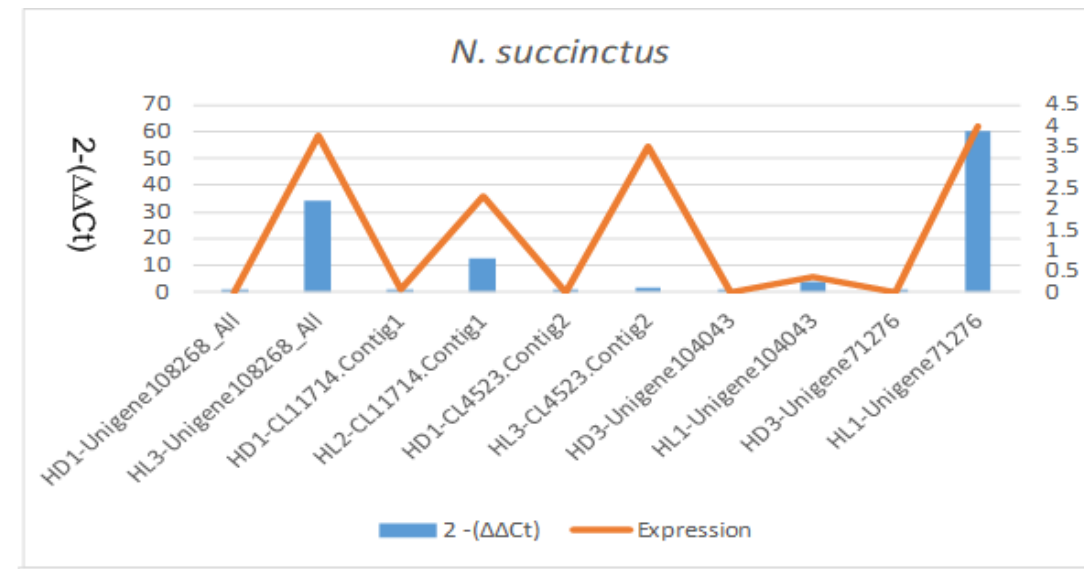

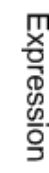

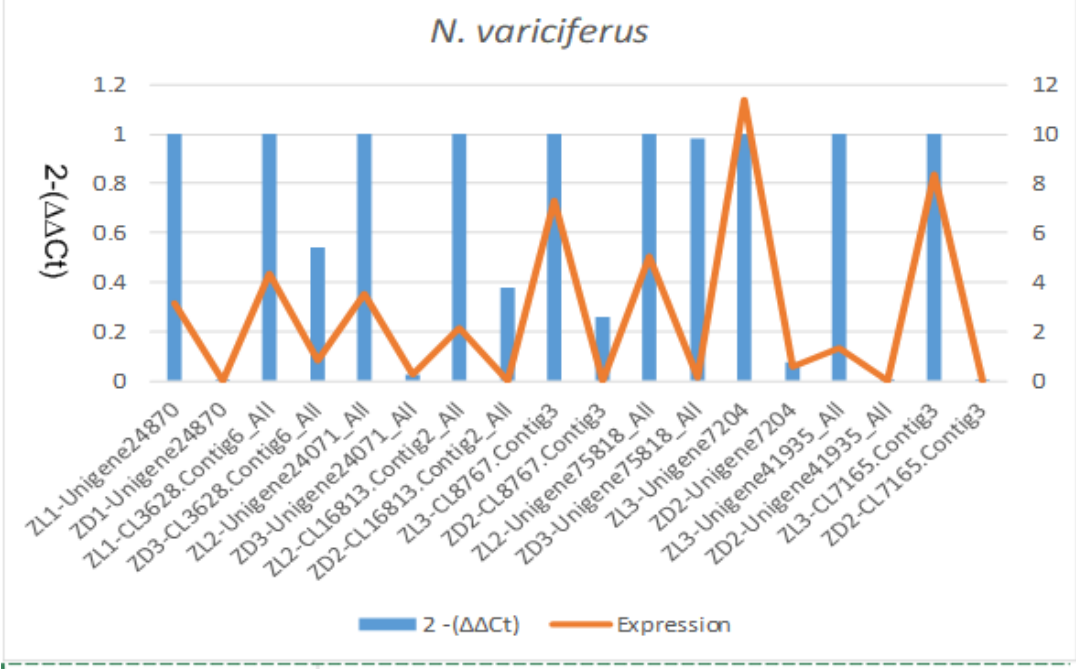

Unigene41935_All Vitamin D metabolism Vitamin D 25-hydroxylase CL3628.Contig6_All Coagulation factor

Unigene75818_All Histone-lysine N-methyltransferase

Unigene108268_All flocculation protein related with oligomeric mucus

Unigene24071_All myosin binding subunit

CL16813.Contig2_All palmitoyltransferase and poly(A) RNA polymerase 
Species name

Rattus norvegicus

Takifugu pardalis

Drosophila melanogaster

Loligo opalescens

Aplysia california

Bdelloura candisda

Cyanea caplillata

Mya areneria sensitive

Mya areneria resistant

Homo sapidens

Tetraonon nigroviridis-a

Tetraonon nigroviridis- $b$

Takifugu rubripes

Chlamys farreri

Crassostrea gigas

Solemya velum

Patinopecten yessoensis

N. succinctus (TTX-resistance)
Domain II

Domain III

RVLCGEWIET VATFKGWMDI

R I L C GEWIET I ATFKGWMDI

$R \vee L C G E W I E S \quad V A T F K G W I Q I$

$R \vee L C G E W I E S$ VATFKGWINI

RVLCGEWIES VATYKGWIDI

RVL CGEYIES VATFKGWTDI

RI L CGKWIEP TATLEGWFEE

RVLCGEWIES VATYKGWI DI

R VLCGEWI DS VATYKGWI DI

RI L C GEWI ET VATFKGWMDI

R I L C GEWIEN I ATFKGWTA I

R VLCGE WI DT VATFKGWME I

RVLCGEWIES VATFKGWTDI

$R \vee L C G E W I E S \quad V A T Y K G W T V I$

RVLCGEWI QS VATY GWIEV

R VLCGEWI QS VATYKGWIEI

$R \vee L C G E W I E S \quad V A T Y K G W T V I$

RVLCGEWIEL VATFEGWIEI 